\title{
The 12-Month Course of ICD-11 Adjustment Disorder in the Context of Involuntary Job Loss
}

\author{
Louisa Lorenz $^{\text {a }}$ (D) , Andreas Maercker ${ }^{\mathrm{a}}$ (1) , Rahel Bachem ${ }^{\mathrm{a}}$
}

[a] Department of Psychology, University of Zurich, Zurich, Switzerland.

Clinical Psychology in Europe, 2020, Vol. 2(3), Article e3027, https://doi.org/10.32872/cpe.v2i3.3027

Received: 2019-05-10 • Accepted: 2020-07-18 • Published (VoR): 2020-09-30

Handling Editor: Cornelia Weise, Philipps-University of Marburg, Marburg, Germany

Corresponding Author: Louisa Lorenz, Department of Psychology, University of Zurich, Binzmuehlestrasse 14/17, CH-8050 Zurich, Switzerland. Tel.: +41 4463574 57. E-mail: 1.lorenz@psychologie.uzh.ch

Supplementary Materials: Materials [see Index of Supplementary Materials]

\begin{abstract}
Background: After its redefinition in ICD-11, adjustment disorder (AjD) comprises two core symptom clusters of preoccupations and failure to adapt to the stressor. Only a few studies investigate the course of $\mathrm{AjD}$ over time and the definition of six months until the remission of the disorder is based on little to no empirical evidence. The aim of the present study was to investigate the course of $\mathrm{AjD}$ symptoms and symptom clusters over time and to longitudinally evaluate predictors of AjD symptom severity.

Method: A selective sample of the Zurich Adjustment Disorder Study, $\mathrm{N}=105$ individuals who experienced involuntary job loss and reported either high or low symptom severity at first assessment (t1), were assessed $\mathrm{M}=3.4(\mathrm{SD}=2.1)$ months after the last day at work, and followed up six ( $\mathrm{t} 2$ ) and twelve months ( $\mathrm{t} 3$ ) later. They completed a fully structured diagnostic interview for AjD and self-report questionnaires.

Results: The prevalence of AjD was $21.9 \%$ at $\mathrm{t} 1,6.7 \%$ at $\mathrm{t} 2$, and dropped to $2.9 \%$ at $\mathrm{t} 3$. All individual symptoms and symptom clusters showed declines in prevalence rates across the three assessments. A hierarchical regression analysis of symptoms at $\mathrm{t} 3$ revealed that more symptoms at the first assessment $(\beta=0.32, p=.002)$ and the number of new life events between the first assessment and $\mathrm{t} 3(\beta=0.29, \mathrm{p}=.004)$ significantly predicted the number of AjD symptoms at $\mathrm{t} 3$.

Conclusion: Although prevalence rates of $\mathrm{AjD}$ declined over time, a significant proportion of individuals still experienced $\mathrm{AjD}$ symptoms after six months. Future research should focus on the specific mechanisms underlying the course of AjD.
\end{abstract}

\section{Keywords}

adjustment disorder, ICD-11, job loss, prevalence, disorders specifically associated with stress 


\section{Highlights}

- Symptoms of ICD-11 adjustment disorder were highly prevalent among individuals who experienced involuntary job loss up to nine months previously.

- In $30 \%$ of the adjustment disorder cases the symptoms persisted beyond the 6month remission threshold defined in the diagnostic manuals.

- Subsequent life events might complicate recovery from adjustment disorder.

- Mechanisms underlying symptom improvement or exacerbation need to be further studied.

The new description of adjustment disorder (AjD) in the International Classification of Diseases, $11^{\text {th }}$ version (ICD-11) includes the presence of (a) one or a series of psychosocial stressor(s); of (b) preoccupation with the stressor(s); of (c) failure to adapt to the stressor(s); and of (d) significant impairment in personal, family, social, educational, occupational or other important areas in functioning (World Health Organisation [WHO], 2018). In contrast, the Diagnostic and Statistical Manual of Mental Disorders, $5^{\text {th }}$ version (DSM-5) does not define specific symptoms and the diagnosis of AjD is not applicable in the presence of any other mental disorder (American Psychiatric Association [APA], 2013). The usage of AjD based primarily on an exclusion criterion in DSM-5 and earlier ICD-versions has resulted in its usage as a diagnostic rest category with subsyndromal character (Bachem \& Casey, 2018; Baumeister \& Kufner, 2009). Another difference between the current manuals is that the DSM-5 distinguishes subtypes of AjD, such as depressed mood, anxiety, disturbance of conduct and mixed subtypes (APA, 2013), whereas the ICD-11 does not.

The diagnostic manuals state that the symptoms usually emerge within one (ICD-11) and three (DSM-5) months after the onset of the stressor and that they typically resolve within 6 months, unless the stressor persists for a longer duration (WHO, 2018). This makes AjD a disorder with an essential benign outcome and spontaneous remission by definition. A few studies that investigated readmission rates for $\mathrm{AjD}$ cases in clinical settings support this concept (Jäger, Burger, Becker, \& Frasch, 2012; Jones, Yates, \& Zhou, 2002). However, AjD is also associated with an elevated risk for concurrent or subsequent mental disorders and for suicidality (Casey \& Doherty, 2012; Gradus et al., 2010; O'Donnell et al., 2016) and the definition of the 6-months' period is still based on little to no empirical evidence. In injury survivors, $16 \%$ of the participants still met the diagnostic criteria of DSM-5 AjD after twelve months post-injury (O’Donnell et al., 2016). In a representative sample from Germany, a significant proportion of individuals who reported AjD symptoms (72\%) indicated that the symptoms were present for six to twenty-four months (Maercker et al., 2012). Finally, a study assessing AjD symptoms several years after organ transplantations found that the time since the medical procedure was 
unrelated to AjD symptom severity (Bachem, Baumann, \& Köllner, 2019). To the best of our knowledge, these are the only studies that specifically focused on the course of AjD over time based on a recent definition of the disorder, all of them putting the six months' period in question.

The Zurich Adjustment Disorder Study (ZADS) investigates the validity of the new ICD-11 definition of AjD in a sample of individuals who involuntarily lost their job and explores predictors of AjD development over time. Previous analyses revealed that the prevalence of $\mathrm{AjD}$ in this high-risk sample was $15.5 \%$ when applying the full ICD-11 diagnostic criteria to a structured diagnostic interview schedule (Perkonigg, Lorenz, \& Maercker, 2018). Based on questionnaire results, the prevalence of a tentative $\mathrm{AjD}$ diagnosis was $25.6 \%$ at approximately three months after the last day at work (Lorenz, Perkonigg, \& Maercker, 2018b), and 18.2\% six months later (Lorenz, Makowski, \& Maercker, 2019).

Demographic factors such as higher age, female gender or low household budget as well as characteristics of the stress experience such as first job loss, a job that required "brainwork", a job with high responsibility, and a larger number of job applications written to get a new position correlated with higher symptom severity and/or higher odds for a diagnosis of AjD (Perkonigg et al., 2018). Established intrapersonal resources that support coping with adversity such as high self-efficacy and sense of coherence were similarly related to fewer symptoms of AjD (Perkonigg et al., 2018). Finally, the socio-interpersonal framework model for stress-response syndromes (Maercker \& Horn, 2013) suggests that different levels of social contexts play a crucial role in the recovery after stress experiences. These contexts include social affects (e.g., shame, anger, loneliness), interactions in close relationships (e.g., social support, empathy) or societal and cultural factors (e.g. social acknowledgement). In accordance with the model, lower self-efficacy, stronger feelings of loneliness, higher dysfunctional disclosure, less perceived social support, and more negative social interactions were identified as correlates of higher symptom severity (Lorenz, Perkonigg, \& Maercker, 2018b).

The aim of the present paper is to expand upon previous findings of the ZADS and other longitudinal investigations. First, we aimed to report on the development of AjD symptoms and ICD-11 core symptom clusters in the context of involuntary job loss across three assessments. Based on the current disorder model and previous research, we expected that the prevalence rates of symptoms and symptom clusters would be high initially and that they would decline after six and twelve months. Second, several potential predictors of $\mathrm{AjD}$ development were explored. We hypothesized that $\mathrm{AjD}$-related features (initial AjD symptoms, life events experienced), socio-demographic factors (gender, age, household income), and psychosocial factors relevant for stress-response syndromes (e.g., personal beliefs, interpersonal resources) would be associated with long-term outcome. 


\section{Method}

\section{Participants and Procedure}

The data for the present analysis stem from the ZADS investigating the new proposal for adjustment disorder in ICD-11 in a sample of individuals who experienced involuntary job loss (Perkonigg et al., 2018). The Ethics Committee of the University of Zurich approved the study in June 2015 and all participants gave written informed consent. The study included all participants who were assessed at three time points with a fully structured clinical diagnostic interview for $\mathrm{AjD}$. The first assessment took place up to nine months after the last day at work (t1), followed by a six-months (t2) and a twelve-months (t3) follow-up assessment. The participants were recruited through local employment offices, newspaper articles, and mailing lists in the greater Zurich area. Participants were excluded if they did not speak German fluently, were unable to give written informed consent, or suffered from a severe mental illness. The latter criterion led to the exclusion of one individual who was assumed to experience a psychotic episode. All participants were invited to participate in the first and second assessment of the study. Since a comparison of extreme groups was planned for the original study, only a sub-sample was invited to the third assessment. Inclusion in the sub-sample was determined after completion of $\mathrm{t} 2$. In the symptomatic group, we invited individuals who (a) met the criteria for an $\mathrm{AjD}$ at $\mathrm{t} 1$ or a subclinical $\mathrm{AjD}$ (either only preoccupation or only failure to adapt) at $\mathrm{t} 1$ and who (b) identified the same worst event at $\mathrm{t} 1$ and $\mathrm{t} 2$. In the non-symptomatic group, we invited individuals who reported a maximum of one symptom of $\mathrm{AjD}$ at $\mathrm{t} 1$ and at $\mathrm{t} 2$. Of the 330 individuals that participated in the first assessment, 294 took part in the second assessment. Of these individuals, 78 met the criteria for the symptomatic group and could be assessed a third time; 27 individuals met the criteria for the non-symptomatic group and could be assessed a third time. This led to a total sample size of $N=105$ for the present analysis. The participant flow is shown in Figure 1.

Table 1 displays a summary of the demographic characteristics of the sample. T1 was conducted $M=3.4(S D=2.1)$ months after the last day at work $(M d n=3.2)$. The interval between $\mathrm{t} 1$ and $\mathrm{t} 3$ was $M=12.3(S D=0.8)$ months. At $\mathrm{t} 3,17.1 \%(n=18)$ of the participants had started a new job since $\mathrm{t} 2,48.6 \%(n=51)$ of the sample continued the new job they had started between $\mathrm{t} 1$ and $\mathrm{t} 2$, 30.5\% $(n=32)$ were still unemployed, and $1.9 \%(n=2)$ experienced a new job loss. 


\section{Figure 1}

Participant Flow of the Zurich Adjustment Disorder Study

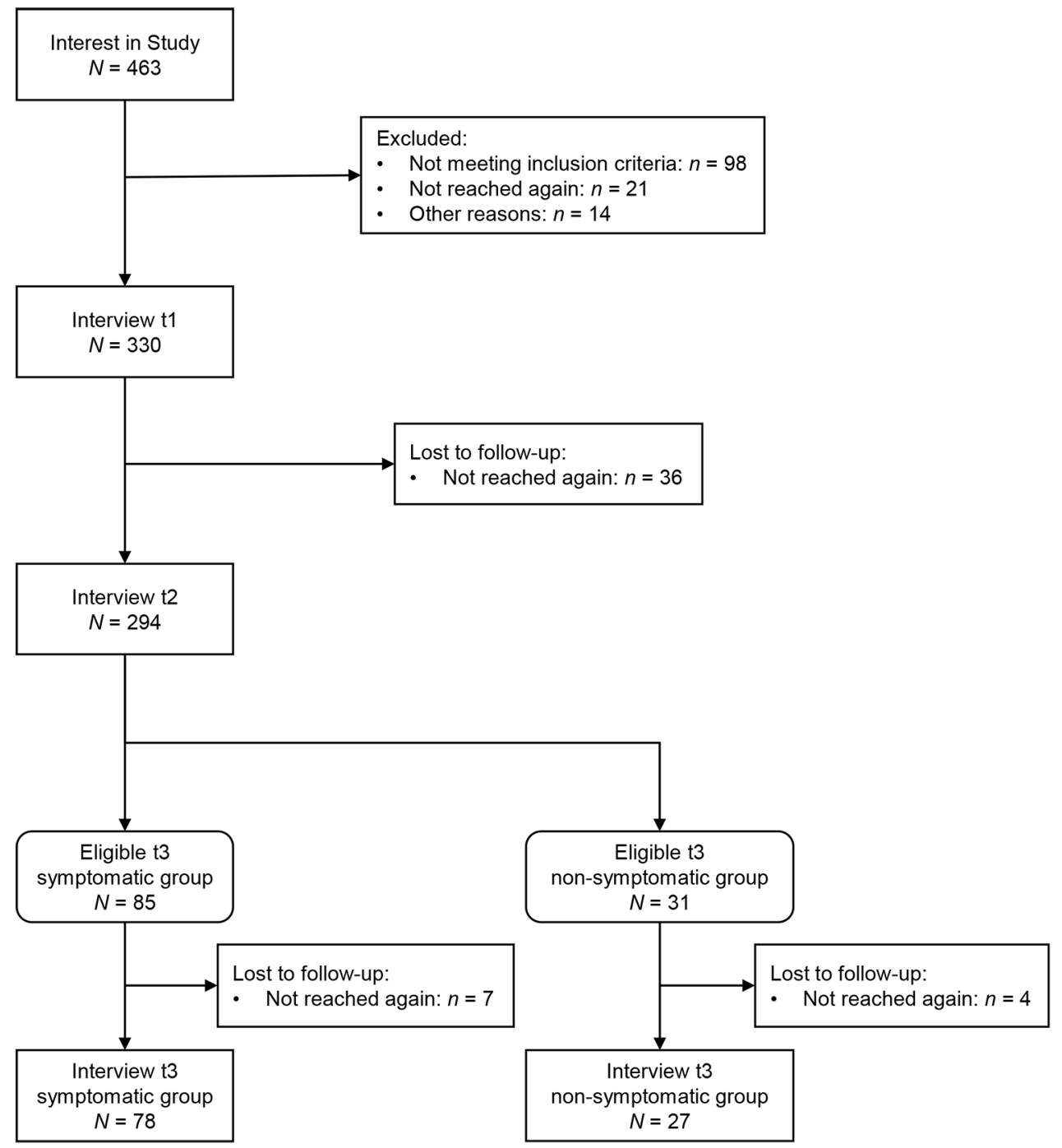

Note. $\mathrm{t} 1=$ first assessment; $\mathrm{t} 2=$ second assessment; $\mathrm{t} 3=$ third assessment. 


\section{Table 1}

Demographic Characteristics of the Sample $(N=105)$

\begin{tabular}{|c|c|c|c|c|}
\hline Variable & $M$ & $S D$ & $N$ & $\%$ \\
\hline Age at t1 & 46.3 & 10.0 & & \\
\hline \multicolumn{5}{|l|}{ Gender } \\
\hline Male & & & 56 & 53.3 \\
\hline Female & & & 49 & 46.7 \\
\hline \multicolumn{5}{|l|}{ Marital status at t1 } \\
\hline Married & & & 38 & 36.2 \\
\hline Separated / divorced & & & 21 & 20.0 \\
\hline Never married & & & 45 & 42.9 \\
\hline Registered partnership & & & 1 & 1.0 \\
\hline Children at $\mathbf{t 1}$ & 0.9 & 1.1 & & \\
\hline \multicolumn{5}{|l|}{ Vocational qualification } \\
\hline On-the-job-training & & & 3 & 2.9 \\
\hline Formal apprenticeship & & & 39 & 37.1 \\
\hline University / University of applied sciences & & & 56 & 53.3 \\
\hline $\mathrm{PhD}$ & & & 3 & 2.9 \\
\hline No qualification & & & 2 & 1.9 \\
\hline Missing & & & 2 & 1.9 \\
\hline
\end{tabular}

\section{Measures}

\section{Adjustment Disorder Module for Composite International Diagnostic Interview (AjD-CIDI)}

Adjustment disorder was assessed with a new module of the Composite International Diagnostic Interview (CIDI) that specifically focuses on AjD after ICD-11 and DSM-5 (AjD-CIDI) (Perkonigg, Strehle, et al., in press). In the beginning, the AjD-CIDI assesses stressors (e.g. family conflict, financial problems, illness of a loved one) that occurred during the 12 months prior to the interview and event-specific characteristics (e.g. time of onset, duration). At the end of this first part, the participants were asked which of the events they experienced as the most distressing. The second part of the interview asks for a range of symptoms occurring in response to this event following the ICD-11 and the DSM-5 definition. The 25 symptoms represent the areas of preoccupation with the stressor and failure to adapt to the stressor, as well as accessory symptoms of avoidance, depression, anxiety and impulsivity. The third part of the module assesses information about onset, recency of symptoms and functional impairment (Perkonigg, Strehle, et al., in press).

We used a modified follow-up version of the AjD module for $\mathrm{t} 2$. In this version, the first part asks for new life events and the most distressing event from the previous 
interview is coded. The participant then indicated the currently most distressing event out of the new and the old events. Then, the second and third part of the AjD-CIDI were applied with regard to the event coded at $\mathrm{t} 1$. At $\mathrm{t} 3$, the symptomatic group was interviewed with a version that asked specifically for symptoms in response to the event they talked about at $\mathrm{t} 1$ and $\mathrm{t} 2$.

The diagnosis of AjD according to ICD-11 (WHO, 2018) was made if the following criteria were met: A) occurrence of a significant life event; B) presence of at least one symptom of preoccupation (recurrent involuntary thoughts about the event, and constant worries related to the event); C) presence of at least two failure to adapt symptoms (concentration problems, difficulties at work/daily activities, loss of interest in work, social network or leisure activities, sleep problems, and loss of self-confidence); D) frequency of symptoms at least 10-15 times per month or clinical relevance of symptoms (impairment at least "moderate" or contact with a health professional about the symptoms); E) exclusion of cases who presented with a current depressive episode and of cases who presented with a current generalized anxiety disorder as defined by the CIDI.

\section{Scales for Predictor Variables}

The General Self-Efficacy Scale (GSE; Schwarzer \& Jerusalem, 1999) was used for the assessment of self-efficacy. The 10-item scale has a 4-point Likert scale response-format $(1$, not correct -4 , absolutely correct). The total score is obtained by summing up all individual items and higher scores indicate higher self-efficacy. The psychometric properties of the GSE were satisfactory in earlier validation studies with internal consistencies of .80 - .90 (Hinz, Schumacher, Albani, Schmid, \& Brähler, 2006; Schwarzer \& Jerusalem, 1999). The internal consistency in the present study was $\alpha=.88$.

We measured sense of coherence using the Sense of Coherence Scale - Revised (SOC-R; Bachem \& Maercker, 2018). The scale, consisting of 13 items, measures manageability, reflection, and balance. The response-format is a 5-point Likert scale (1, not at all, - 5, completely). All items are summed up to build a total score of the SOC-R, with one recoded item. Higher scores indicate a higher sense of coherence. Earlier validation studies reported satisfactory psychometric properties for the SOC-R with internal consistencies of $\alpha=.75-.81$ (Bachem \& Maercker, 2018; Mc Gee, Höltge, Maercker, \& Thoma, 2018). The internal consistency in the present study was $\alpha=.71$.

A composite score of two single items from other scales was used to measure feelings of loneliness (Lorenz, Perkonigg, \& Maercker, 2018b). We used one item from the Brief Symptom Inventory - 18 (Spitzer et al., 2011) and one item of the Social Functioning Questionnaire (Tyrer et al., 2005). The item formulations were 'How strong did you experience feelings of loneliness during the past 7 days?' and 'I feel lonely and isolated from other people'. The response-format was a 5-point Likert scale (0, not at all - 4, very strong) and a 4-point Likert scale (0, almost all the time - 3, not at all), respectively. The 
latter item was recoded before building a sum score with the first item of the scale. The correlation between the two items in the present study was $\alpha=.70$.

The Disclosure of Trauma Questionnaire (DTQ) was used in an abbreviated form (Pielmaier \& Maercker, 2011) to measure dysfunctional disclosure. The scale, consisting of 12 items with a 6-point Likert scale ( 0 , not at all - 5, absolutely) response-format, measures the urge to talk, the reluctance to talk, and emotional reactions while disclosing. The individual items are summed up to build a total score; higher scores indicate higher dysfunctional disclosure. Previous studies found satisfying psychometric properties for the DTQ (Müller, Beauducel, Raschka, \& Maercker, 2000; Müller \& Maercker, 2006). The internal consistency of the abbreviated form was $\alpha=.75$ in previous studies (Pielmaier \& Maercker, 2011) and $\alpha=.81$ in the present study.

We used the Social Support Questionnaire, short form (FSozU-K; Fydrich, Sommer, Tydecks, \& Brähler, 2009) to measure perceived social support. The 14 items are answered on a 5-point Likert scale (1, don't agree, - 5, agree). The mean of all answered items is used to build the total score and higher scores indicate higher perceived social support. The psychometric properties in the validation of the FSozU-K were satisfactory with an internal consistency of $\alpha=.94$ (Fydrich et al., 2009). The internal consistency in the present study was $\alpha=.93$.

A subset of items of the Daily Hassles Scale (Perkonigg \& Wittchen, 1998) was used to measure negative social interactions (Lorenz, Perkonigg, \& Maercker, 2018b). Six items measured negative interactions with the partner, children, parents, siblings, friends, or neighbours during the last two weeks. The original 4-point Likert scale response-format of the items (1, often - 4, never) was reverse coded, so that a higher mean score indicates more negative social interactions. The internal consistency was $\alpha=.68$ in a previous study (Lorenz, Perkonigg, \& Maercker, 2018b) and $\alpha=.73$ in the present study.

The Social Acknowledgement Questionnaire (SAQ; Maercker \& Müller, 2004) measured perceived acknowledgement of the difficult situation of the individual by the social environment. The 16 items, answered on a 4-point Likert scale ( 0 , not at all - 3, complete$l y$ ), measure general disapproval, disapproval by family or friends, and recognition as a victim. Following the authors of the scale, the total score was built by summing up items 3, 9, and 11 through 16, and subtracting items 1, 2, 4 through 8, and 10. A higher score indicates more social acknowledgement. The validation study of the questionnaire reported satisfactory psychometric properties with an internal consistency of $\alpha=.86$ (Maercker \& Müller, 2004). The internal consistency in the present study was $\alpha=.73$.

\section{Statistical Analysis}

Data were analysed using SPSS version 23. The highest number of missing values was found for social acknowledgement (13\%), all other variables had less than $3 \%$ missing values and data were missing completely at random. Pairwise case deletion was used in the analyses. The prevalence of ICD-11 AjD was computed with and without consideration 
of the exclusion criterion. To investigate predictive factors, we performed a hierarchical regression analysis with the number of symptoms at $\mathrm{t} 3$ as outcome. We decided to include all symptoms that were measured by the AjD-CIDI to increase the variance of the outcome variable and because there is still uncertainty about the best conceptualisation of AjD (Lorenz, Hyland, Perkonigg, \& Maercker, 2018). The analysis included three steps. In the first step, we included the number of symptoms at $t$, the total number of life events reported at $\mathrm{t} 1$, and the total number of new life events reported between $\mathrm{t} 1$ and t3 as predictors. The second step included socio-demographic characteristics (gender, age, household income $<4000$ Swiss francs) and the third step included psychosocial variables (general self-efficacy, loneliness, dysfunctional disclosure, perceived social support, negative social interactions, social acknowledgement). In the second and third step, we included predictor variables that were found to be associated with initial symptom severity and 6-months outcomes in previous publications from this sample (Lorenz, Hyland, et al., 2018; Lorenz, Perkonigg, \& Maercker, 2018a, 2018b; Perkonigg et al., 2018). The final model was selected based on the significance of the F-statistics. No multicollinearity was found based on the VIF measure (ranged between 1.030 and 1.078).

\section{Results}

\section{Descriptives}

The total amount of symptoms as measured by the AjD-CIDI was $M=7.1(S D=5.5$; $M d n=7.0$, range $=0-19)$ at $\mathrm{t} 1, M=4.3(S D=5.0 ; M d n=2.0$, range $=0-20)$ at $\mathrm{t} 2$, and $M=$ $2.1(S D=2.8 ; M d n=1.0$, range $=0-13)$ at $\mathrm{t} 3$. The total number of life events reported at $\mathrm{t} 1$ was $M=2.3(S D=1.2$, range $=1-7)$ and the total number of new life events experienced between $\mathrm{t} 1$ and $\mathrm{t} 3$ was $M=1.0(S D=1.3$, range $=0-7)$. The majority of participants $(74.3 \%)$ indicated the job loss, financial problems or problems with authorities as their worst event at $\mathrm{t} 1$, followed by family matters (22.9\%; family conflicts/separation/illness or death of family member). The descriptive statistics for the predictor variables and the correlation coefficients between the main predictor variables can be found in the supplementary material.

\section{Prevalence of AjD Symptoms}

The prevalence rates of the individual symptoms as measured by the AjD-CIDI are displayed in Figure 2. For the majority of symptoms, the prevalence was highest at $\mathrm{t} 1$ and lowest at $\mathrm{t} 3$. The symptoms measuring preoccupation with the stressor, sleep disturbances (as part of failure to adapt), and feeling low and sad (as part of depressive symptoms) were the most prevalent at $\mathrm{t} 1$ with over $40 \%$ of the individuals reporting each of them. At $\mathrm{t} 2$, repetitive thoughts, feeling low and sad, and feeling discouraged and hopeless for the future (depressive symptom) were the most prevalent symptoms 
(each over $30 \%$ ). The most prevalent symptoms at $\mathrm{t} 3$ were repetitive thoughts, rumination about the event, and avoiding situations or individuals that could remind of the event (avoidance symptom) with roughly a $20 \%$ prevalence each.

\section{Figure 2}

Prevalence (\%) of Individual Symptoms That May Occur in ICD-11 Adjustment Disorder Across the Three Assessments

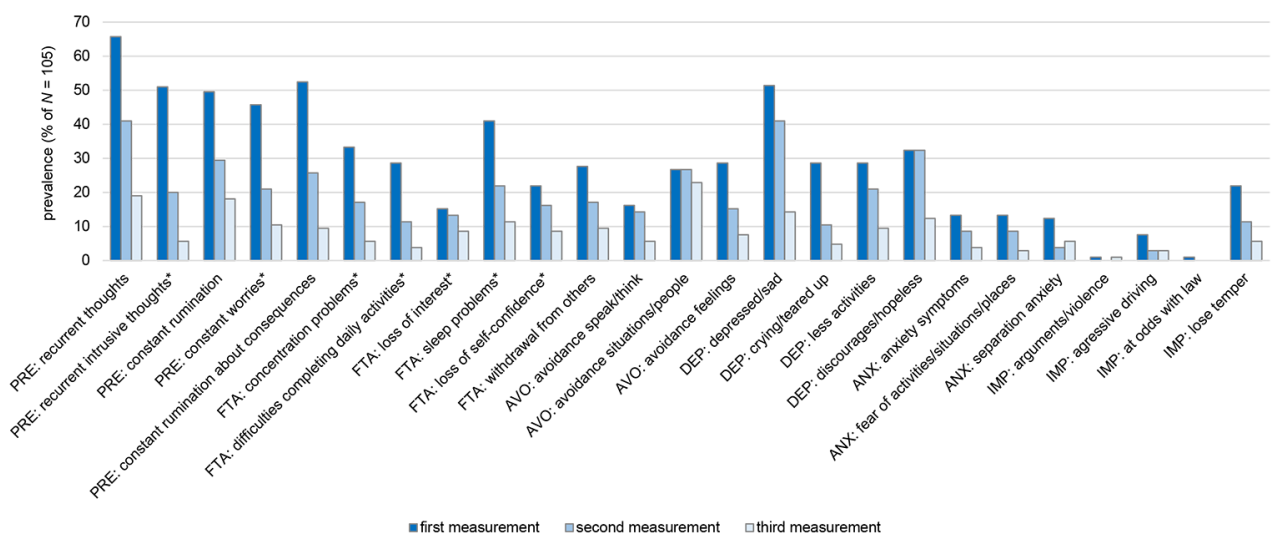

Note. $\mathrm{PRE}=$ Preoccupation; FTA = Failure to adapt $\mathrm{AVO}=$ Avoidance $; \mathrm{DEP}=$ Depression; $\mathrm{ANX}=$ Anxiety; IMP = Impulsivity.

*items used for diagnostic algorithm for adjustment disorder.

\section{Prevalence of AjD Symptom Groups}

Table 2 displays the prevalence of the diagnostic criteria across the three assessments. Criterion A was met by every participant since the presence of a stressor was an inclusion criterion of the study. The prevalence rates of preoccupation (Criterion $\mathrm{B}$ ), failure to adapt (Criterion C), and impairment in social functioning (Criterion D) were highest for the first assessment and declined over time. The prevalence rate of exclusive disorders (Criterion E) remained stable across the three assessments. Approximately every fifth individual met the full diagnostic criteria at $\mathrm{t} 1$ (21.9\%). This prevalence declined to $6.7 \%$ at $\mathrm{t} 2$, and to $2.9 \%$ at $\mathrm{t} 3$. The majority of individuals reported no AjD across all assessments ( $n=80 ; 76.2 \%$ ). Most of the other participants met the diagnostic guidelines only at $\mathrm{t} 1$ $(n=16,15.2 \%)$ or only at $\mathrm{t} 1$ and $\mathrm{t} 2(n=5,4.8 \%)$. One individual $(1.0 \%)$ received an AjD diagnosis at all three assessments. 
Table 2

Prevalence of Adjustment Disorder Criteria Across the Three Assessments

\begin{tabular}{|c|c|c|c|c|c|c|}
\hline \multirow[b]{2}{*}{ Adjustment Disorder Criterion } & \multicolumn{2}{|c|}{ T1 } & \multicolumn{2}{|c|}{$\mathbf{T} 2$} & \multicolumn{2}{|c|}{ T3 } \\
\hline & $N$ & $\%$ & $N$ & $\%$ & $N$ & $\%$ \\
\hline Criterion A: Event & 105 & 100.0 & 105 & 100.0 & 105 & 100.0 \\
\hline Criterion B: Preoccupation & 63 & 60.0 & 32 & 30.5 & 15 & 14.3 \\
\hline Criterion C: Failure to adapt & 44 & 41.9 & 18 & 17.1 & 10 & 9.5 \\
\hline Criterion D: Impairment & 82 & 78.1 & 67 & 63.8 & 40 & 38.1 \\
\hline Criterion E: Exclusive disorders & 10 & 9.5 & 10 & 9.5 & 9 & 8.6 \\
\hline ICD-11 Adjustment disorder without exclusion criterion & 29 & 27.6 & 12 & 11.4 & 4 & 3.8 \\
\hline ICD-11 Adjustment disorder with exclusion criterion & 23 & 21.9 & 7 & 6.7 & 3 & 2.9 \\
\hline
\end{tabular}

Note. $\mathrm{T} 1$ = first assessment; $\mathrm{T} 2$ = second assessment; T3 = third assessment.

\section{Prediction of AjD Symptoms at $\mathbf{t} 3$}

Table 3 displays the results of the hierarchical regression analysis for the total number of AjD-CIDI symptoms at $\mathrm{t}$. The first step included the number of AjD-CIDI symptoms at $t 1$, the number of life events reported at $t 1$, and the number of new live events experienced between $\mathrm{t} 1$ and $\mathrm{t} 3$ as predictors. This model was significant, $F(3,86)=7.648$, $p<.001$. The second model, which included socio-demographic characteristics, and the third model, which included psycho-social variables, did not significantly increase the fit of the model. Thus, the model only including adjustment disorder related characteristics (Model 1) was interpreted. A higher number of AjD-CIDI symptoms at $\mathrm{t} 1$ and a higher number of life events experienced between $\mathrm{t} 1$ and $\mathrm{t} 3$ were associated with a higher number of AjD-CIDI symptoms at $\mathrm{t} 3$. The model explained $18 \%$ of the variance in the outcome (adjusted $R^{2}=.183$ ).

Table 3

Hierarchical Regression Results (Standardized $\beta$ Coefficients) for the Total Number of AjD-CIDI Symptoms at the Third Assessment $(N=105)$

\begin{tabular}{|c|c|c|c|}
\hline \multirow[b]{2}{*}{ Predictor } & \multicolumn{3}{|c|}{ Model } \\
\hline & 1 & 2 & 3 \\
\hline Number of AjD-CIDI symptoms at $\mathrm{t} 1$ & $0.316^{* *}$ & $0.365^{* * *}$ & $0.278^{*}$ \\
\hline Number of life events at $t 1$ & 0.060 & 0.083 & 0.088 \\
\hline Number of new life events between $t 1$ and $t 3$ & $0.291^{* *}$ & $0.286^{* *}$ & $0.292^{* *}$ \\
\hline Gender & & $-0.235^{*}$ & -0.205 \\
\hline Age (t1) & & 0.046 & 0.007 \\
\hline Household income < 4000 SFr (t1) & & 0.000 & -0.001 \\
\hline General self-efficacy (t1) & & & -0.079 \\
\hline
\end{tabular}




\begin{tabular}{|c|c|c|c|}
\hline \multirow[b]{2}{*}{ Predictor } & \multicolumn{3}{|c|}{ Model } \\
\hline & 1 & 2 & 3 \\
\hline Sense of coherence (t1) & & & -0.029 \\
\hline Loneliness (t1) & & & 0.164 \\
\hline Dysfunctional disclosure (t1) & & & -0.032 \\
\hline Perceived social support (t1) & & & 0.078 \\
\hline Negative social interactions ( $\mathrm{t} 1$ ) & & & 0.069 \\
\hline Social acknowledgement (t2) & & & -0.035 \\
\hline$F$ & $7.648^{* * *}$ & 2.130 & 0.518 \\
\hline$R^{2}$ & .211 & .267 & .300 \\
\hline adjusted $R^{2}$ & .183 & .214 & .181 \\
\hline$\Delta R^{2}$ & & .056 & .033 \\
\hline
\end{tabular}

Note. Gender: 1 = male; 2 = female; Household income $<4000$ SFr $(0=$ no; $1=$ yes $)$. ${ }^{*} p<.05 .{ }^{* *} p<.01 .{ }^{* * *} p<.001$.

\section{Discussion}

The aim of the present analysis was to investigate the course of adjustment disorder in the context of involuntary job loss over the course of twelve months. It was the first investigation of prevalence rates according to ICD-11 with a new structured diagnostic interview in a high-risk sample. We found an $\mathrm{AjD}$ prevalence rate of $21.9 \%$ at the first assessment. Previous studies using ICD-10 or DSM-IV criteria found prevalence rates ranging between $6.9 \%$ and 38\% in high risk populations (e.g., Mitchell et al., 2017; Rundell, 2006), between 3\% and 12\% in medical settings (e.g., Fernández et al., 2012; Yaseen, 2017), and between $11 \%$ and $17 \%$ in psychiatric settings (Bruffaerts, Sabbe, \& Demyttenaere, 2004; Shear et al., 2000). Based on a self-report questionnaire, studies investigating the new ICD-11 approach reported varying prevalence rates between $21 \%$ and 61\% in high-risk populations (e.g., Dannemann et al., 2010; Dobricki, Komproe, de Jong, \& Maercker, 2010). However, they refer to a tentative diagnosis and did not apply the ICD-11 exclusion criterion. The prevalence rate in this sample, consisting of extreme groups with high or low AjD symptoms at previous assessments, dropped to $3 \%$ at the third assessment, which is only slightly higher than prevalence rates found in general population-based samples (e.g., Ayuso-Mateos et al., 2001; Glaesmer, Romppel, Braehler, Hinz, \& Maercker, 2015). At the same time, the prevalence rate was lower than the twelve-months prevalence rate found in the O'Donnell et al. (2016) study investigating the DSM-5 model in a post-injury sample. This could be either an effect of the different diagnostic guidelines applied (ICD-11 or DSM-5) or an effect of the stressor (job loss vs. injuries). Future studies should aim at a direct comparison between ICD-11 and DSM-5 diagnostic guidelines. 
As expected, there was a decline in AjD symptoms over time. This generally supports the assumption of a favourable outcome of AjD. However, a substantial proportion (seven of the twenty-three cases) with an $\mathrm{AjD}$ at the first assessment still met the diagnostic criteria for an $\mathrm{AjD}$ six months later. This represents $30 \%$ of the $\mathrm{AjD}$ cases that show a longer duration of the disorder than the conditional six-month threshold in ICD-11 and DSM-5. It could be argued that the life event 'job loss', which was rated to be the worst event by the majority of the sample, or its consequences is often not resolved within the time period of six months the ICD-11 mentions as "typical" for a resolution. This argument is supported by the high number of new or subsequent life events in the present sample, which might complicate recovery. It emphasizes the difficulty of applying time period features like six months in stress-related disorders and implies to use this feature only after a thorough substantive examination and a flexible interpretation of the abovementioned period.

The second aim of this study was to investigate factors that predict $\mathrm{AjD}$ symptoms after twelve months. The hierarchical approach allowed us to examine whether only AjD-related characteristics explain long-term outcome or whether socio-demographic factors and psychosocial processes add explanatory power over the course of twelve months. The results indicate that higher initial symptomatology and more life stressors following the event significantly predicted higher symptomatology twelve months later and that AjD-related characteristics might be a sufficient explanation for symptom severity over the course of twelve months, supporting the concept of a stress-response syndrome. However, the selection of potential risk and protective factors was limited, and future studies should include other relevant predictors since the model was only able to explain $18 \%$ of the variation in symptom severity after twelve months.

We included socio-demographic and psychosocial predictors that were associated with initial symptom severity in earlier studies (e.g., Lorenz, Perkonigg, \& Maercker, 2018b; Perkonigg et al., 2018). Although these predictors were not longitudinally associated with AjD symptoms, they were associated with initial symptom severity. Since initial symptom severity was one of the strongest predictors of long-term outcome, the effect of the socio-demographic and psychosocial predictors on $\mathrm{t} 3 \mathrm{AjD}$ symptoms could be indirect, via symptoms at $\mathrm{t} 1$. Hence, future studies could focus on a possible mediation effect of initial symptom severity on the association between socio-demographic and psychosocial predictors and long-term outcome. If this mediation was true, it could be reasonable to target these factors to achieve a better long-term outcome. This assumption finds support in two recent self-help intervention studies for AjD. These interventions aimed at enhancing resilience for example by improving problem-solving skills or mobilizing social support and showed medium to large effect sizes for the reduction of AjD related symptomatology over time (Bachem \& Maercker, 2016; Eimontas, Rimsaite, Gegieckaite, Zelviene, \& Kazlauskas, 2018). Alternative explanations for the result that especially the number of life events predicted symptom severity at $\mathrm{t} 3$ could be memory 
effects or attention deficits. The AjD-CIDI stressor list also covers psychosocial stress of minor intensity, such as troubles with neighbours or giving up a hobby. Individuals who are worse off could be particularly sensitive to these minor stressors while better adjusted individuals may find it unnecessary to report these events.

The analyses for $\mathrm{AjD}$ symptoms were based on all symptoms that may occur in $\mathrm{AjD}$ rather than only the ICD-11 core symptom cluster of preoccupation and failure to adapt because of the differences between the major diagnostic classification systems. While the ICD-11 defines specific core symptoms (WHO, 2018), the DSM-5 kept the previous definition that is not based on specific criteria but on the exclusion of other mental disorders (APA, 2013). These dissimilarities are a result of the lack of research around AjD and of a lack of agreement on the main characteristics of the disorder, and they might result in differences in access to treatment. Across the three assessments, different symptoms of preoccupation with the stressor were among the most prevalent symptoms, supporting the inclusion of this symptom group in the diagnostic guidelines in ICD-11. Symptoms that reflect depressive reactions were also commonly present, suggesting that it might be reasonable to include mood alterations in the AjD definition as it is the case in DSM-5. These results could be a first evidence for the validity of both approaches and further revisions of the guidelines might include features of both definitions. Future research should not only focus on the most prevalent symptoms but also try to identify symptoms that are associated with high functional impairment or that show high discriminatory power.

The use of the new ICD-11 diagnostic guidelines and a fully structured clinical diagnostic interview make this study unique. Still, it has several limitations. First, the data stems from a particular high-risk sample, which limits the generalizability to all AjD cases. Second, the sample for this study was based on specific selection criteria. We specifically defined a symptomatic and a non-symptomatic group to increase variance in the data. Moreover, we lifted inclusion criterion b) for the non-symptomatic group in order to be able to investigate incidence rates for adjustment disorder. This specific methodology complicated interpretation of prevalence findings at $t 3$. Furthermore, the recruitment was based on self-selection since we did not apply a systematic or stratified recruitment strategy. These methodological concerns restrict the generalizability of the results to the whole population of unemployed individuals. Third, we did only control for the presence of a depressive episode and/or generalised anxiety disorder and not the full list of exclusive disorders as recommended by ICD-11. Future studies should consider the full range of clinically meaningful exclusions. Fourth, the interval between assessments was chosen at six months to investigate the proposal of the diagnostic guidelines for AjD. Research that includes shorter intervals between assessments could shed further light into the dynamics of the disorder. Last, the number of predictors in the hierarchical regression could have limited the power of the analysis considering the sample size. This could have masked some predictive effects and future studies should increase the sample 
size. In addition, loneliness was assessed with two items from different scales rather than with an established questionnaire.

Adjustment disorder has been a diagnostic category that received little attention in research despite a frequent use in clinical practice (Evans et al., 2013; Reed, Correia, Esparza, Saxena, \& Maj, 2011). The relatively high prevalence of AjD in this study, the methodological concerns raised by our findings, and the aforementioned issues of disorder definition again stress the importance of a systematic inclusion of AjD in research in order to understand maladaptive responses to life stress better, especially since AjD is associated with a higher risk for the development of severe psychopathology and suicidality (e.g., Casey \& Doherty, 2012; O’Donnell et al., 2016). This study furthermore showed that even though AjD symptomatology shows a favourable course over time, it can also persist beyond the six-month threshold as proposed by ICD-11 and DSM-5. Further research is needed to understand the mechanisms underlying the disorder and determining the long-term outcome of AjD. Moreover, future studies comparing prevalence rates between ICD-11 and DSM-5 may deepen our understanding of maladjustment to stressful life events.

\footnotetext{
Funding: This research was funded by a grant of the Swiss National Science Foundation (\#100019_159436) and financial support by the Jacobs Foundation.
}

Competing Interests: The authors have declared that no competing interests exist.

Acknowledgments: This work is part of the Zurich Adjustment Disorder Study (2014-2018). We thank co-PI Dr. Axel Perkonigg, all respondents of the study for their participation and Lisa Makowski, BSc., for her work regarding data collection and data processing. We acknowledge the Office of Economy and Labour Zurich for cooperation on respondents' recruitment and Dr. Beesdo-Baum, Dr. Wittchen and Dipl. math. Jens Strehle (TU Dresden) for collaboration on the AjD-CIDI module.

Ethics Approval: The authors assert that all procedures contributing to this work comply with the ethical standards of the relevant national and institutional committees on human experimentation and with the Helsinki Declaration of 1964 and its later amendments. All persons gave their written informed consent prior to their inclusion in the study.

Data Availability: Data from this study are not publicly available as informed consent and ethical approval for public data sharing were not obtained from participants. The data are readily available upon request by qualified scientists. Any enquiries regarding data accessibility can be addressed to the first author.

\section{Supplementary Materials}

The Supplementary Materials contain the descriptive statistics of the main measures of the study and the correlations between study variables (for access see Index of Supplementary Materials below). 


\section{Index of Supplementary Materials}

Lorenz, L., Maercker, A., \& Bachem, R. (2020). Supplementary materials to "The 12-month course of ICD-11 adjustment disorder in the context of involuntary job loss" [Descriptive statistics and correlation coefficients]. PsychOpen. https://doi.org/10.23668/psycharchives.3463

\section{References}

American Psychiatric Association. (2013). Diagnostic and statistical manual of mental disorders (5th ed.). Arlington VA, USA: Author. https://doi.org/10.1176/appi.books. 9780890425596.744053

Ayuso-Mateos, J. L., Vazquez-Barquero, J. L., Dowrik, C., Lehtinen, V., Dalgard, O. S., Casey, P., .. . Wilkinson, G. (2001). Depressive disorders in Europe: Prevalence figures from the ODIN study. The British fournal of Psychiatry, 179, 308-316. https://doi.org/10.1192/bjp.179.4.308

Bachem, R., Baumann, J., \& Köllner, V. (2019). ICD-11 adjustment disorder among organ transplant patients and their relatives. International fournal of Environmental Research and Public Health, 16, Article 3030. https://doi.org/10.3390/ijerph16173030

Bachem, R., \& Casey, P. (2018). Adjustment disorder: A diagnose whose time has come. fournal of Affective Disorders, 227, 243-253. https://doi.org/10.1016/j.jad.2017.10.034

Bachem, R., \& Maercker, A. (2016). Self-help interventions for adjustment disorder problems: A randomized waiting-list controlled study in a sample of burglary victims. Cognitive Behaviour Therapy, 45, 397-413. https://doi.org/10.1080/16506073.2016.1191083

Bachem, R., \& Maercker, A. (2018). Development and psychometric evaluation of a revised sense of coherence scale. European Journal of Psychological Assessment, 34, 206-215.

https://doi.org/10.1027/1015-5759/a000323

Baumeister, H., \& Kufner, K. (2009). It is time to adjust the adjustment disorder category. Current Opinion in Psychiatry, 22, 409-412. https://doi.org/10.1097/YCO.0b013e32832cae5e

Bruffaerts, R., Sabbe, M., \& Demyttenaere, K. (2004). Attenders of a university hospital psychiatric emergency service in Belgium. Social Psychiatry and Psychiatric Epidemiology, 39, 146-153. https://doi.org/10.1007/s00127-004-0708-x

Casey, P., \& Doherty, A. (2012). Adjustment disorder: Implications for ICD-11 and DSM-5. The British Journal of Psychiatry, 201, 90-92. https://doi.org/10.1192/bjp.bp.112.110494

Dannemann, S., Einsle, F., Kämpf, F., Joraschky, P., Maercker, A., \& Weidner, K. (2010). Anpassungsstörungen nach einem neuen diagnostischen Konzept bei Patienten einer psychosomatischen Poliklinik - Beschwerden, Veränderungsbereitschaft und Psychotherapiemotivation [New diagnostic concept of adjustment disorders in psychosomatic outpatients - Symptom severity, willingness to change, psychotherapy motivation]. Zeitschrift für Psychosomatische Medizin und Psychotherapie, 56, 231-243. https://doi.org/10.13109/zptm.2010.56.3.231

Dobricki, M., Komproe, I. A., de Jong, J., \& Maercker, A. (2010). Adjustment disorders after severe life-events in four postconflict settings. Social Psychiatry and Psychiatric Epidemiology, 45, 39-46. https://doi.org/10.1007/s00127-009-0039-z 
Eimontas, J., Rimsaite, Z., Gegieckaite, G., Zelviene, P., \& Kazlauskas, E. (2018). Internet-based selfhelp intervention for ICD-11 adjustment disorder: Preliminary findings. The Psychiatric Quarterly, 89, 451-460. https://doi.org/10.1007/s11126-017-9547-2

Evans, S. C., Reed, G. M., Roberts, M. C., Esparza, P., Watts, A. D., Correia, J. M., . . Saxena, S. (2013). Psychologists' perspectives on the diagnostic classification of mental disorders: Results from the WHO-IUpsyS Global Survey. International fournal of Psychology, 48, 177-193. https://doi.org/10.1080/00207594.2013.804189

Fernández, A., Mendive, J. M., Salvador-Carulla, L., Rubio-Valera, M., Luciano, J. V., Pinto-Meza, A., ... Serrano-Blanco, A. (2012). Adjustment disorder in primary care: Prevalence, recognition and use of services. The British fournal of Psychiatry, 201, 137-142. https://doi.org/10.1192/bjp.bp.111.096305

Fydrich, T., Sommer, G., Tydecks, S., \& Brähler, E. (2009). Fragebogen zur sozialen Unterstützung (F-SozU): Normierung der Kurzform (K-14) [Social Support Questionnaire (F-SozU): Standardization of short form (K-14)]. Zeitschrift für Medizinische Psychologie, 18, 43-48.

Glaesmer, H., Romppel, M., Braehler, E., Hinz, A., \& Maercker, A. (2015). Adjustment disorder as proposed for ICD-11: Dimensionality and symptom differentiation. Psychiatry Research, 229, 940-948. https://doi.org/10.1016/j.psychres.2015.07.010

Gradus, J. L., Qin, P., Lincoln, A. K., Miller, M., Lawler, E., \& Lash, T. L. (2010). The association between adjustment disorder diagnosed at psychiatric treatment facilities and completed suicide. Journal of Clinical Epidemiology, 2, 23-28. https://doi.org/10.2147/CLEP.S9373

Hinz, A., Schumacher, J., Albani, C., Schmid, G., \& Brähler, E. (2006). Bevölkerungsrepräsentative Normierung der Skala zur Allgemeinen Selbstwirksamkeitserwartung [Standardization of the general self-efficacy scale in the German population]. Diagnostica, 52, 26-32.

https://doi.org/10.1026/0012-1924.52.1.26

Jäger, M., Burger, D., Becker, T., \& Frasch, K. (2012). Diagnosis of adjustment disorder: Reliability of its clinical use and long-term stability. Psychopathology, 45, 305-309.

https://doi.org/10.1159/000336048

Jones, R., Yates, W. R., \& Zhou, M. H. (2002). Readmission rates for adjustment disorders: Comparison with other mood disorders. Journal of Affective Disorders, 71, 199-203. https://doi.org/10.1016/S0165-0327(01)00390-1

Lorenz, L., Hyland, P., Perkonigg, A., \& Maercker, A. (2018). Is adjustment disorder unidimensional or multidimensional? - Implications for ICD-11. International fournal of Methods in Psychiatric Research, 27, Article e1591. https://doi.org/10.1002/mpr.1591

Lorenz, L., Makowski, L., \& Maercker, A. (2019). Diagnostik und Risikofaktoren der ICD-11 Anpassungsstörung: Ergebnisse der Zürcher Studie zu Anpassungsstörungen nach ungewolltem Arbeitsplatzverlust [Diagnostics and risk factors for ICD-11 adjustment disorders - Findings from the Zurich Adjustment Disorder Study]. Trauma \& Gewalt, 13, 6-18. https://doi.org/10.21706/tg-13-1-6 
Lorenz, L., Perkonigg, A., \& Maercker, A. (2018a). The course of adjustment disorder following involuntary job loss and its predictors of latent change. Clinical Psychological Science, 6, 647-657. https://doi.org/10.1177/2167702618766290

Lorenz, L., Perkonigg, A., \& Maercker, A. (2018b). A socio-interpersonal approach to adjustment disorder: The example case of involuntary job loss. European fournal of Psychotraumatology, 9, Article 1425576. https://doi.org/10.1080/20008198.2018.1425576

Maercker, A., Forstmeier, S., Pielmaier, L., Spangenberg, L., Brähler, E., \& Glaesmer, H. (2012). Adjustment disorders: Prevalence in a representative nationwide survey in Germany. Social Psychiatry and Psychiatric Epidemiology, 47, 1745-1752.

https://doi.org/10.1007/s00127-012-0493-x

Maercker, A., \& Horn, A. B. (2013). A socio-interpersonal perspective on PTSD: The case for environments and interpersonal processes. Clinical Psychology \& Psychotherapy, 20, 465-481. https://doi.org/10.1002/cpp.1805

Maercker, A., \& Müller, J. (2004). Social acknowledgment as a victim or survivor: A scale to measure a recovery factor of PTSD. Fournal of Traumatic Stress, 17, 345-351. https://doi.org/10.1023/B:JOTS.0000038484.15488.3d

Mc Gee, S. L., Höltge, J., Maercker, A., \& Thoma, M. V. (2018). Evaluation of the revised Sense of Coherence scale in a sample of older adults: A means to assess resilience aspects. Aging \& Mental Health, 22, 1438-1447. https://doi.org/10.1080/13607863.2017.1364348

Mitchell, A. J., Sheth, B., Gill, J., Yadegarfar, M., Stubbs, B., Yadegarfar, M., \& Meader, N. (2017). Prevalence and predictors of post-stroke mood disorders: A meta-analysis and meta-regression of depression, anxiety and adjustment disorder. General Hospital Psychiatry, 47, 48-60. https://doi.org/10.1016/j.genhosppsych.2017.04.001

Müller, J., Beauducel, A., Raschka, J., \& Maercker, A. (2000). Kommunikationsverhalten nach politischer Haft in der DDR - Entwicklung eines Fragebogens zum Offenlegen der Traumaerfahrungen [Communication after political imprisonment: Disclosure of the traumatic experiences]. Zeitschrift fur Politische Psychologie, 8, 413-427.

Müller, J., \& Maercker, A. (2006). Disclosure und wahrgenommene gesellschaftliche Wertschätzung als Opfer als Prädiktoren von PTB bei Kriminalitätsopfern [Disclosure and perceived social acknowledgement as victim as PTSD predictors in crime victims]. Zeitschrift für Klinische Psychologie und Psychotherapie, 35, 49-58. https://doi.org/10.1026/1616-3443.35.1.49

O’Donnell, M. L., Alkemade, N., Creamer, M., McFarlane, A. C., Silove, D., Bryant, R., . . Forbes, D. (2016). A longitudinal study of adjustment disorder after trauma exposure. The American Journal of Psychiatry, 173, 1231-1238. https://doi.org/10.1176/appi.ajp.2016.16010071

Perkonigg, A., Lorenz, L., \& Maercker, A. (2018). Prevalence and correlates of ICD-11 adjustment disorder: Findings from the Zurich Adjustment Disorder Study. International fournal of Clinical and Health Psychology, 18, 209-217. https://doi.org/10.1016/j.ijchp.2018.05.001

Perkonigg, A., Strehle, J., Lorenz, L., Maercker, A., \& Beesdo-Baum, K. (in press). Reliability and validity of the ICD-11 adjustment disorder diagnosis according to a new module for the Composite International Diagnostic Interview. fournal of Traumatic Stress. 
Perkonigg, A., \& Wittchen, H. U. (1998). The Daily-Hassles Scale: Research version. München, Germany: Max-Planck-Institut für Psychiatrie.

Pielmaier, L., \& Maercker, A. (2011). Psychological adaptation to life-threatening injury in dyads: The role of dysfunctional disclosure of trauma. European fournal of Psychotraumatology, 2, Article 8749. https://doi.org/10.3402/ejpt.v2i0.8749

Reed, G. M., Correia, J. M., Esparza, P., Saxena, S., \& Maj, M. (2011). The WPA-WHO Global Survey of Psychiatrists' Attitudes Towards Mental Disorders Classification. World Psychiatry: Official Journal of the World Psychiatric Association (WPA), 10, 118-131. https://doi.org/10.1002/j.2051-5545.2011.tb00034.x

Rundell, J. R. (2006). Demographics of and diagnosis in Operation Enduring Freedom and Operations Iraqi Freedom personell who were psychiatrically evacuated from the theater of operations. General Hospital Psychiatry, 28, 352-356.

https://doi.org/10.1016/j.genhosppsych.2006.04.006

Schwarzer, R., \& Jerusalem, M. (1999). Skalen zur Erfassung von Lehrer- und Schülermerkmalen. Dokumentation der psychometrischen Verfahren im Rahmen der Wissenschaftlichen Begleitung des Modellversuchs Selbstwirksame Schulen [Scales for the assessment of teacher and student characteristics - Documentation of the psychometric procedures of the scientific evaluation of the model project self-efficacious schools]. Berlin, Germany: Freie Universität Berlin.

Shear, M. K., Greeno, C., Kang, J., Ludewig, D., Frank, E., Swartz, H. A., \& Hanekamp, M. (2000). Diagnosis of nonpsychotic patients in community clinics. The American fournal of Psychiatry, 157, 581-587. https://doi.org/10.1176/appi.ajp.157.4.581

Spitzer, C., Hammer, S., Löwe, B., Grabe, H. J., Barnow, S., Rose, M., . . Franke, G. H. (2011). Die Kurzform des Brief Symptom Inventory (BSI -18): erste Befunde zu den psychometrischen Kennwerten der deutschen Version [The Short Version of The Brief Symptom Inventory (BSI-18): Preliminary psychometric properties of the German translation]. Fortschritte der Neurologie · Psychiatrie, 79, 517-523. https://doi.org/10.1055/s-0031-1281602

Tyrer, P., Nur, U., Crawford, M., Karlsen, S., MacLean, C., Rao, B., \& Johnson, T. (2005). The Social Functioning Questionnaire: A rapid and robust measure of perceived functioning. International Journal of Social Psychiatry, 51, 265-275. https://doi.org/10.1177/0020764005057391

World Health Organisation. (2018). International Classification of Diseases, 11th revision. Retrieved from https://icd.who.int/browse11/1-m/en

Yaseen, Y. A. (2017). Adjustment disorder: Prevalence, sociodemographic risk factors, and its subtypes in outpatient psychiatry clinic. Asian fournal of Psychiatry, 28, 82-85. https://doi.org/10.1016/j.ajp.2017.03.012 


\section{EACLIPT}

Clinical Psychology in Europe (CPE) is the official journal of the European Association of Clinical Psychology and Psychological Treatment (EACLIPT).

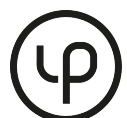

leibniz-psychology.org

PsychOpen GOLD is a publishing service by Leibniz Institute for Psychology Information (ZPID), Germany. 